\title{
Charge Densities above Pulsar Polar Caps
}

\author{
A. Jessner \\ Max-Planck-Institut für Radioastronomie, Auf dem Hügel 69, \\ D-53121Bonn, Germany \\ H. Lesch and Th. Kunzl \\ Institut für Astronomie und Astrophysik, Universität München, \\ Scheinerstr. 1, D-81679 München, Germany
}

A simplified model provided the framework for our investigation into the distribution of energy and charge densities above the polar caps of a rotating neutron star. We assumed a neutron star with $m=1.4 M_{\odot}, r=10 \mathrm{~km}$, dipolar field $\left|B_{0}\right|=10^{12} \mathrm{G}, B|| \Omega$ and $\Omega=2 \pi \cdot(0.5 \mathrm{~s})^{-1}$. The effects of general relativity were disregarded. The induced accelerating electric field $E_{\| \mid}$reaches $E_{0}=2.5 \cdot 10^{13} \mathrm{~V} \mathrm{~m}^{-1}$ at the surface near the magnetic poles. The current density along the field lines has an upper limit $n_{G J}$, when the electric field of the charged particle flow cancels the induced electric field: At the poles $n_{\mathrm{GJ}}\left(r=r_{\mathrm{ns}}, \theta=0\right)=1.4 \cdot 10^{17} \mathrm{~m}^{-3}$. The work function (surface potential barrier) $E_{\mathrm{W}}$ is approximated by the Fermi energy $E_{\mathrm{F}}$ of magnetised matter. Following Abrahams and Shapiro (1992) one needs to revise the surface density from the canonical $1.4 \cdot 10^{8} \mathrm{~kg} \mathrm{~m}^{-3}$ down to $\rho_{\mathrm{Fe}}=2.9 \cdot 10^{7} \mathrm{~kg} \mathrm{~m}^{-3}$. With $E_{\mathrm{F}}\left(\rho_{\mathrm{Fe}}\right)=\frac{2 \cdot \pi^{4} \hbar^{4} \mathrm{c}^{2}}{e^{2} B^{2} m_{e}} \cdot\left(\frac{\rho_{\mathrm{Fe}} \cdot(28-2)}{56 \cdot m_{\mathrm{p}}}\right)^{2}$ we obtain a value of $E_{\mathrm{F}}=E_{\mathrm{W}}=417 \mathrm{eV}$. There are two relevant particle emission processes: Field (cold cathode) emission by quantum-mechanical tunneling of charges through the surface potential

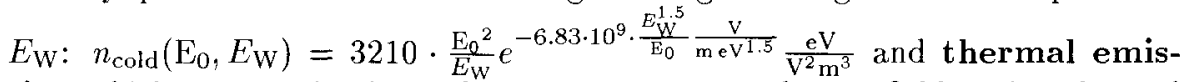
sion which is a purely classical process. In strong electric fields it is enhanced by the lowering of the potential barrier due to the Schottky effect. The combined Dushman-Schottky equation $n_{\mathrm{DS}}\left(E_{0}, E_{\mathrm{W}}, T\right)=\frac{A_{0}}{e \cdot c} \cdot T^{2} \cdot e^{-\frac{E_{\mathrm{W}}}{k T}+\frac{e}{k T} \cdot \sqrt{\frac{e \cdot E_{0}}{4 \pi \epsilon_{0}}}}$ with $A_{0}=\frac{4 \pi \cdot m_{e} \cdot e \cdot k^{2}}{h^{3}}=120.2 \mathrm{Acm}^{-2}$ tells us, that at temperatures $>2 \cdot 10^{5} \mathrm{~K}$ the the Goldreich-Julian current can be supplied thermal emission alone. The surface temperature however has a lower limit in the order of $10^{5} \mathrm{~K}$ due to the rotational braking. Therefore, in most cases a sufficient supply of charges for the Goldreich-Julian current is available and the electrical field accelerating the particles will be quenched as a result of their abundance. Otherwise a residual equilibrium electric field $E_{\text {eq }}$ remains with: $E_{\text {eq }}=E_{0} \cdot\left(1-\frac{n_{e}}{n_{\mathrm{GJ}}}\right)$ and hence the equilibrium density is: $n=n_{\text {field }}\left(\mathrm{E}_{\text {eq }}, E_{\mathrm{W}}\right)+n_{\mathrm{DS}}\left(\mathrm{E}_{\text {eq }}, E_{\mathrm{W}}, T\right)$ For a temperature just below the onset of thermal emission $\left(T=1.85 \cdot 10^{5} \mathrm{~K}\right)$ the charge density is found to vary almost linearly with the work function $E_{\mathrm{W}}$ for values of $E_{\mathrm{W}}$ between 0.3 and $2 \mathrm{keV}$. At the chosen value for $E_{\mathrm{W}}$ of $417 \mathrm{eV}$ the residual electric field amounts to only $8.5 \%$ of the vacuum value. Even in the residual electric field the particles are rapidly accelerated to relativistic energies balanced by inverse Compton and curvature radiation losses. 


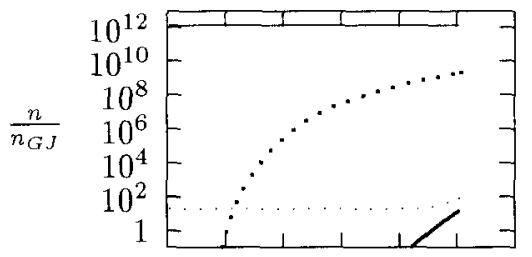

$\begin{array}{lllllllllll}0.1 & 0.2 & 0.4 & 0.6 & 0.8 & 1.0 & 1.2\end{array}$ $\mathrm{T}\left(10^{6} \mathrm{~K}\right)$

We computed the radiation density $U$ along the parameterised field lines $r\left(\theta, \theta_{0}\right)$ as given by the differential equation: $\frac{\mathrm{d} U}{\mathrm{~d} \theta}=$ $-4 U \cdot \frac{r_{n s}^{2}}{r\left(\theta, \theta_{0}\right)^{3}}-n \cdot m_{\mathrm{e}} \cdot c^{2} \frac{\mathrm{d} \gamma}{\mathrm{d} \theta}$. From this we obtain the local temperature of the radiation bath: $T_{\text {eff }}=\left(\frac{3 U}{a}\right)^{1 / 4}$ and the Lorentz factor obeys the differential equation: $\frac{\mathrm{d} \gamma}{\mathrm{d} \theta}=\left.\frac{\mathrm{d} \gamma}{\mathrm{d} \theta}\right|_{\mathrm{E}}+\left.\frac{\mathrm{d} \gamma}{\mathrm{d} \theta}\right|_{\text {curvRad }}+$ Fig.1: emissivities $n / n_{\mathrm{GJ}}$ for different $\left.\frac{\mathrm{d} \gamma\left(T_{\mathrm{eff}}\right)}{\mathrm{d} \theta}\right|_{\text {inv Compt }}$. Fig. 2 shows a numer-
$E_{\mathrm{W}}$ and as a function of the surface tem- ical solution of the deqns.: a neutron star perature. thin solid: emission at $E_{\mathrm{W}}=$ with a surface temperature of $\mathrm{T}=2 \cdot 10^{5} \mathrm{~K}$ $417 \mathrm{eV}$, thick dots: total emissivity for and $n=0.9 \cdot n_{\mathrm{GJ}}$ forms a hot "shell" with $E_{\mathrm{W}}=417 \mathrm{eV}$ and $0.01 \mathrm{E}_{0}$, thin dots: total emissivity for $E_{\mathrm{W}}=2065 \mathrm{eV}$, thick a maximum temperature of $T_{\text {eff }}=2.9 \cdot 10^{6}$ solid: total emissivity for $E_{\mathrm{W}}=2065 \mathrm{eV} \mathrm{K}$ and a $\gamma$ of $1.1 \cdot 10^{8}$ at a height of 130 and $0.1 \mathrm{E}_{0}$, $\mathrm{km}$ above the surface, which would be a strong source of $X$ and $\gamma$-rays. Pair production is often assumed to be an inevitable occurrence at the poles where it would create a dense neutral plasma

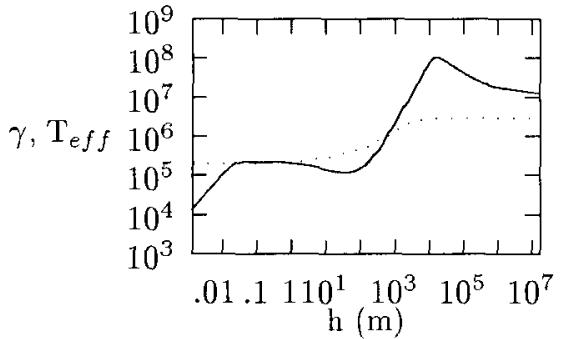

Fig.2: Lorentz factor $\gamma$ (solid) and effective temperature $T_{\text {eff }}$ (dotted) for a neutron star with a surface temperature of $\mathrm{T}=2 \cdot 10^{5} \mathrm{~K}$ and $n=0.9 \cdot n_{\mathrm{G} J}$ in a particle avalanche ("inner gap"). Our computations verify the existence of a pair production zone $200 \mathrm{~m}$ above the pulsar surface only if inverse Compton braking were ignored, otherwise the local pair creation probability never rises above zero. We have thus found two principal scenarios that exhaustively cover the set of field lines on a polar cap: The "Saturated" magnetosphere is expected around most rent is established and $E_{\|}=0$.

Although $\gamma$ is expected to be 1 , instabilities producing mildly relativistic particles are possible. Neutron stars surrounded by a gaseous atmosphere can liberate charges even more easily and are therefore always expected to have a magnetosphere of this type. A "Starved" magnetosphere would be expected on cold neutron stars which show X-ray emissions from their relativistic magnetosphere with very high values of $\gamma$. The Lorentz factors are however too large to explain pulsar radio emissions.

Conclusion: Inner gap pair production is ruled out in both scenarios and hence for all pulsars. These findings together with the observations of radio pulsars with $\mathrm{X}$-ray temperatures $>10^{6} \mathrm{~K}$ cast further doubt on the validity of the basic assumptions ( $n \gg n_{\mathrm{GJ}}$ at $h>h_{\mathrm{gap}}$ ) behind standard pulsar models.

Acknowledgements. We are indebted to S. Ahnert, Sidney Sussex College, Cambridge for his help with the presentation.

\section{References}

A. Abrahams and S. Shapiro, The Structure and Evolution of Neutron Stars, Ed. D. Pines, R. Tamagaki and S. Tsuruta, Addison Wesley, 210, (1992) 